\title{
Reactive powder concrete based on multicomponent cement systems with multilevel optimization of the disperse composition
}

\author{
Evgeniy Velichko and Ekaterina Shokodko* \\ Moscow State University of Civil Engineering, Yaroslavskoe shosse, 26, Moscow, 129337, Russia
}

\begin{abstract}
This paper study the process of multilevel optimization of particulate structure of concrete. The improvement of performance characteristics of concrete can be achieved with a minimum binder content. Theoretical and experimental principles for the design of an optimal structure of concrete with an increased concentration of solid phase and operational characteristics, with a complex organo-mineral additive are developed on the basis of a rational proportion of chemical and finely dispersed mineral components. The high density of the structure is achieved by obtaining the dense packages by applying strong particles and grains with high elastic modulus and optimal parameters (dispersion and content) for each structural level. It is shown that it is expedient to use several structural levels of the particles of the clinker phase, and pozzolanization of the matrix must be carried out in accordance with the optimal formulation. Finely-dispersed slag, microsilica and highly dispersed fraction of cement were considered as mineral components, and superplasticizer of polycarboxylate type was used as a chemical additive.
\end{abstract}

\section{Introduction}

Strength and durability of concrete and reinforced concrete with the minimum consumption of cement are the most urgent problems in up-to-date construction, which require constant development. Multilevel modification of the dispersed phase of the composition is the main method of obtaining high-performance concrete with a minimum binder content and highperformance characteristics, such as Reactive Powder Concrete (RPC) [1,2]. The basic principle of obtaining RPC is the creation of a dense homogeneous structure, provided by the optimization of the granulometric composition at various structural levels. High concentration of solid phase per unit volume is achieved by obtaining the most dense packing of particles and grains of the cement system with optimal parameters (dispersion and content) for each structural level.

To obtain high strength of RPC, it is necessary to create a discrete-continuous dispersion-granulometric composition of particles and grains of the solid phase. Each subsequent fine-dispersed fraction should be distributed in an optimal amount with the maximum filling of interparticle or intergranular voids between less dispersed particles or

\footnotetext{
Corresponding author: bezuglova-e@inbox.ru
} 
grains. In the creation of such dense packages, the key factor is the choice of elastic, pozzolanic, hydration and other properties of particles and grains for various structural levels. These properties ensure a balanced homogeneous distribution of particles and grains in accordance with electro-kinetic potential and stress state associated with the geometric dimensions and distribution of particles, as well as with variable thermodynamic potentials during hardening. For example, the optimal distance between the particles of the first-level filling fraction in the stressed state is $2 \mathrm{D}$ ( $\mathrm{D}$ is the particle size), and their volume fraction is 0.2 [3]. With the indicated parameters, the dispersed particles are at a considerable distance from each other, the energy fields of their deformation are not superimposed and do not make an appropriate contribution to the stressed state of the material. It is also presented that $95 \%$ of the strain energy associated with the particle and the surrounding matrix is within a spherical volume of radius D from the center of the particle [3]. Thus, particles can be considered as isolated from each other only if the distance between them is greater than $2 \mathrm{D}$ or the volume fraction is less than 0.2. This corresponds to the results of studies of multicomponent cement systems (MCS) with mineral modifiers (MM), the application of which is optimized for the geometric parameters of particles of different structural-energy levels $[4,5]$. In addition, it is necessary to consider that in practical systems of composite structure there is a probability that two or more dispersed particles can unite, and will form a separate aggregate. The probability of combining two and three particles with a content of MM in the MCS in an amount of 50\% is 0.5 and 0.02 , respectively [6,7]. In such microvolumes of cement systems, the pozzolanic reaction almost does not proceed, and they are presented as pseudo pores 5-7 microns in size (the case of three particles) and 0.5-1.5 microns (two particles). These structural defects make a significant contribution to the reduction in strength, frost resistance, deformation characteristics of cementitious matrix; they are distributed in the same way as pairs and triples of dispersed particles. The parameters of the distributions are their content and size.

Obviously, cracks are formed more rapidly in composites with an increased volume fraction of the dispersed phase and the presence of aggregates of these particles. In particular, low performance properties (frost resistance, weather resistance, etc.) of pozzolan portland cement are associated with this phenomenon.

Thus, the volume fraction and the dispersed particle size of each subsequent fraction of the material must correspond to the size of interparticle voids and their volume in the previous one. The volume of the remaining voids in such a system will be minimal. In case of insufficient content of particles of any level to fill the corresponding voids in the previous one, an inhomogeneous structure of cement stone with a high degree of irregularity and with a higher entropy, and also a relatively low strength is obtained. On the contrary, in conditions of increased content of particles - there will be aggregation of particles and, for example, pseudo pore formation in case of using MM.

The choice of materials for the homogeneous distribution of particles at different structural levels with respect to dispersion in order to form a well-ordered microstructure should provide either a hydration process or the pozzolanic reaction in micro-volumes between the particles of neighboring levels. In addition, to ensure the high strength of such systems, dispersed particles of all levels must have a high modulus of elasticity, which will provide a more uniform stress state, including cases of applying energy or agent to the system [3]. The level of allowable energy input to the material in this case increases.

In general, the more dispersed phase either decreases or increases the modulus of elasticity of the matrix phase, depending on the modulus of the dispersed particles. In the work of Paul, et al., using the deformation energy theorems, equations were obtained for determining the modulus of the composite as a function of the moduli of elasticity of the matrix, the dispersed phase, and the volume content of the latter [3]. However, the cracks that can develop during the cooling of the composite below its manufacturing temperature, 
the pores formed during the manufacturing process, and the pseudo-pores formed under stress due to weak coupling over the phase interfaces, lead to significantly lower elastic modules relative to those calculated theoretically. The greatest modulus of elasticity, maximum strength, reduction of cracks and minimum pseudo-pores is ensured by manufacturing a composite with dispersed particles of small size and a more strengthened bond in the contact zone between the matrix and the dispersed particles. Typically, the phase interface during the hydration of clinker minerals, the formation of hydrated phases, and their solidification, proceeding according to the mechanism of molecular selection, are sinks for defects [8]. It is obvious that the modulus of elasticity of cement stone with MM is determined by the modulus of elasticity of the hydrated phases, and also by the modulus of elasticity of the relics of the clinker component particles and MM. The modulus of elasticity of relics of clinker particles is evaluated by values several times higher than those for hydrated phases. Consequently, the most efficient MM for cement systems is a finely dispersed blast-furnace granular slag, whose particles are characterized by high strength and modulus of elasticity and the ability to independent hydraulic hardening. Hydration of slag minerals significantly increases the density and strength of the cement stone, especially in the contact zone with portland cement hydrate phases. The relics of slag contribute significantly to the integral modulus of elasticity and the strength of the synthesized MCS.

Increase in strength parameters is also ensured by limiting the degree of hydration of the clinker minerals while maintaining the maximum volume of non-hydrated phase - relicts of particles with a strength of $310 \mathrm{MPa}$ (the average strength of hydrated phases is $135 \mathrm{MPa}$ ).

Thus, the synthesis of a multi-component cement system with a multilevel dispersiongranulometric modification of the structure is most expedient to implement using the following principal provisions.

From the definition of RPC, a fine aggregate for concrete is taken in two or three fractions [9-11,14]. For example, a coarse fraction of an average of $0.3 \mathrm{~mm}$ in an amount of $80 \%$ and a small fraction of $0.12 \mathrm{~mm}-20 \%$, which provides a significant reduction in its intergranular voids. The volume of the multicomponent binder is ascertained by the absolute volume method, and the dispersed structural levels can be synthesized as follows. For example, the first level is represented by portland cement with a dispersion of $270-350$ $\mathrm{m}^{2} / \mathrm{kg}$ or less in order to ensure a long-term preservation of strong clinker particle relics (with a high modulus of elasticity) in a cement matrix. To fill the first level of interparticle voids of portland cement, finely dispersed blast-furnace granulated slag is used in an amount of $20-25 \%$ with an optimum dispersion of $450-500 \mathrm{~m}^{2} / \mathrm{kg}$ [4]. To fill the second level - highly disperse Portland cement - in amount of $8-12 \%-900-1100 \mathrm{~m}^{2} / \mathrm{kg}[4,7]$ is used, ensuring a uniform pozzolanic reaction in all micro-volumes containing MM and preventing the formation of their aggregates. For the following level- microsilica in amount of $9-12 \%-18000-21000 \mathrm{~m}^{2} / \mathrm{kg}$ is used, which stabilizes the structure of cement stone and fixes hydrate phase particles in the position of near coagulation, increasing its density significantly, and forms strong low-basic hydro-silicate phases $[6,12,15]$.

Studies of finely dispersed slag with optimal parameters showed the homogenous distribution of its particles in the matrix of a multi-component cement stone (determined by the method of scanning electron microscopy with microanalysis). The variation coefficient for the distribution of slag particles was about $0.5 \%$. Such parameters of the components of the first structural level provide a high concentration of solid phase per unit volume of MCS, a uniform proceeding of pozzolanic reaction in the micro-volumes of the matrix, as well as a high level of relics relative to the coarsely dispersed fraction of the clinker component. It should be mentioned that the finely dispersed cement fraction is characterized by a content of particles less than $5.8 \mu \mathrm{m}$ in size in an amount up to $12 \%$, and particles of $-5-30 \mu \mathrm{m}$ in an amount up to $59 \%$ (determined by a laser analyzer), which is approximately twice the content in industrial portland cement. It is obvious that the high 
water demand of concrete synthesized in this way requires the obligatory application of super-plasticizing water-reducing additives of the third generation, in particular, of polycarboxylate type.

Dissolution of the initial phases and separation of the hydrate products from the supersaturated solution is the dominant mechanism at the active stage (2-10 hours after the cement is mixed with water), i.e., there is a recrystallization route along Le Chatelier ("through the solution"). At this stage, finely dispersed clinker particles, mainly of the second filling level of interparticle voids, completely dissolve and redistributed as hydration products in consequence of molecular selection around the adjacent larger particles of the corresponding phases $[5,8,13]$. The layer of hydrosilicate gel becomes thicker in the outer direction, absorbing the rod- and needle-like crystals of ettringite and forming a conglomerate type of microstructure. Hydrosulfoaluminates of calcium within the microstructure of the calcium-silicate hydrate phase contribute to an increase in its density and strength. It is obvious that simultaneously their inclusion as an impurity phase reduces the strength of the cement stone. Thus, the contribution of calcium hydrosulfoaluminates to the strength of the structure of cement stone is of a dual nature. The resulting calcium hydroxide reacts with silicon dioxide of microsilica, with the formation of strong low-basic calcium hydrosilicates, thus accelerating the dissolution of the initial calcium-silicate phases. Most often, these modifications of calcium hydrosilicates are observed in form of fibers, rods, curls (type I). Fibrous particles have a tubular structure formed as a result of coiling of hydrosilicates in the form of a dense low-defect foil. Tubular structures due to capillary forces easily absorb and lose water, determining their increased deformation of shrinkage and swelling, and lower frost resistance. However, due to the high density and low permeability, these properties are improved relatively typical concretes with the same types of MM. This is due to the product formed as a result of the pozzolanic reaction, called by Mikhaelis as "hydrolite". It is characterized by a higher density due to its formation at a lower kinetic energy of the hydrate phase particles and a smaller content (by a factor of 1.5-2) of chemically bound water. By the time when the thickness of the layer of products reaches a value of $0.5-1 \mu \mathrm{m}$, the shells of the neighboring particles of the hydrated calcium-silicate phases of the clinker, slag and products of the pozzolanic reaction begin to grow together, forming strong aggregates and floccules due to the same structure and molecular selection [8]. This corresponds to the end of setting (8-12 hours) and usually the maximum on the differential heat release curve. After this, the growth of the surface of the reaction ceases and the slowdown begins. Almost complete hydration of fine particles of clinker component, high rate of pozzolan reaction between $\mathrm{Ca}(\mathrm{OH})_{2}$ and silicon dioxide of microsilica, activation and partial hydration of mineral slag phases with an increase in the volume of all the formed hydrate phases by an average of 2.2 times relative to the initial products provides a dense cement stone mainly with gel pores.

It should be mentioned that the hydration process (irrespective of the route: recrystallization (A. Le Chatelier), - the topochemical (V.Michaelis)) of clinker phases leads to chemical degradation of its particles, which in essence is the process of corrosion of initial particles with high free Gibbs energy which clinker acquired in the process of its production at a maximum firing temperature of $1450{ }^{\circ} \mathrm{C}$.

Thus, in order to significantly reduce the porosity and increase the concentration of the solid phase in a unit volume, as well as the strength of the cement stone, a dense multicomponent multilevel packing of the matrix particles is required, with a high degree of ordering of the disperse composition, providing, if necessary, a high level of pozzolanic reaction in all micro-volumes. Also, the condition of a low water-cement ratio must be met. Complete hydration of the clinker particles is not required. The relics of the clinker and slag particles, if present, provide a mechanism for self-healing of structure defects formed during the operation of products and structures, integrating part of its high strength into the strength 
and elastic properties of the cement stone. In this case, the contact zone of the hydrated phases and relics of the clinker and slag particles is highly defective, in view of the different types and sizes of their crystal lattices, which are formed topo-chemically in the later periods of hardening.

The most important feature of the topochemical process is that the formation of hydration products is self-stimulated by mechanical stresses that take place when they arise and grow in the surficial region of the relict of the clinker particle. In the topochemical process, part of the chemical energy of the transformation of the calcium-silicate phases of the clinker and slag into the products of hydration is "accumulated" by the relicts of their particles in the form of mechanical deformation energy, stimulating the formation of new hydrate phases. The appearance of each microparticle of hydration products in the surficial region of the relict of a clinker or slag particle is associated with deformation of the crystal matrix around the formed particle. The interatomic distances in hydration products and the relict of the clinker particle are always different, and even small differences in interatomic distances lead to large strains around the formed microparticle $[8,13]$. These stresses are reduced in the hydrate phase by their relaxation due to the amorphous structure and elasticity of the resulting hydration products in the form of calcium hydrosilicates. The described processes in the relics of clinker and slag particles create defective zones and conditions for their further hydration. In the hydrated calcium-silicate phase, the mentioned processes also provided stress relaxation - one of the conditions for formation of a strong cement stone that links its components into a single conglomerate. It is obvious that as the hydration reaction develops into the clinker particle, an integral decrease in the strength of the solid phase of the cement stone takes place. This is due to the strength of the clinker particles $(310 \mathrm{MPa})$ and the products of hydration (135 MPa). However, in time, the strength of cement stone grows according to the logarithmic law. It is associated mainly with a decrease in porosity and a lower degree of disorder in its structure. The volume of hydration products relative to the clinker particles increases by an average of 2.2 times. Pores, being stress concentrators, reduce the strength of brittle bodies by 3 or more times.

Application of multilevel disperse-granulometric modification of concrete allows to solve the problems of obtaining structural materials with new characteristics - strength up to 200 $\mathrm{MPa}$ and above, optimized rates of initial components, high construction and technical properties, incl. deformation characteristics. As a plasticizing-water-reducing component, comb-type additives are applied, they are based on polycarboxylate ether. Due to the steric effect of the grafted side polymer chains, the distance between the particles increases, as a result repulsive forces with $10 \mathrm{~nm}$ for pure water begin to manifest up to $100 \mathrm{~nm}$ or more, creating an energy barrier for coagulation of hydrate phase particles and stabilizing the system at an early stage at low water-cement ratios.

\section{Materials and Experimental Methods}

Experimental studies on the properties of concrete were carried out using the following materials. As a fine aggregate, two fractions of sand were taken from sieves 0,315 and 0,16 $\mathrm{mm}$ in quantities of 80 and 20\%. Portland cement of Belgorod plant grade PC500-D0 with a dispersion of $290 \mathrm{~cm}^{2} / \mathrm{kg}$, finely dispersed slag of Lipetsk metallurgical plant with a specific surface of $450 \mathrm{~m}^{2} \mathrm{~kg}$, microsilica - 18000-21000 $\mathrm{m}^{2} / \mathrm{kg}$ and finely -dispersed cement $890 \mathrm{~cm}^{2} / \mathrm{kg}$ in an amount of $8 \%$ in accordance with the experimental data. The content of multicomponent cement synthesized from these components in optimal amounts was equal to 660,720 and $780 \mathrm{~kg} / \mathrm{m}^{3}$ of concrete. Glenium 430 (OOO BASF-Building Systems) was used as a super-plasticizing water-reducing component in the amount of $0.45 \%$ of cement mass. 


\section{Results and Discussion}

The strength of concrete at the total cementitious material contents of 660,720 and $780 \mathrm{~kg} / \mathrm{m}^{3}$ after hardening under standard conditions at the age of 1 day was 48.3; 57.4; 67.6 MPa, respectively and at the age of 28 days was $144 ; 178 ; 209 \mathrm{MPa}$, respectively.

Derivatographic and X-ray diffraction studies of its samples at the age of 28 days after hardening under standard conditions and heat-moisture treatment were performed to assess the quality of the concrete structure (Table 1).

Table 1. Results of the analysis of thermogravimetric and radiographic curves

\begin{tabular}{|c|c|c|c|c|c|c|}
\hline \multirow[t]{2}{*}{ No. } & \multirow{2}{*}{$\begin{array}{l}\text { Type of mineral modifier } \\
\text { and its content, } \%\end{array}$} & \multicolumn{3}{|c|}{$\begin{array}{c}\text { The temperature, }{ }^{\circ} \mathrm{C} \\
\text { Weight loss, } \%\end{array}$} & \multirow[t]{2}{*}{$\mathrm{Ca}(\mathrm{OH})_{2}$} & \multirow[t]{2}{*}{$\begin{array}{c}\text { Rate of } \\
\text { hydration, } \\
\%\end{array}$} \\
\hline & & 136-147 & $519-531$ & $822-832$ & & \\
\hline \multicolumn{7}{|c|}{ At the age of 28 days after hardening under standard conditions } \\
\hline 1 & Control composition & 19,5 & 25,2 & 31,3 & 24,5 & 55 \\
\hline 2 & Finely-dispersed slag -25 & 14,1 & 21,6 & 25,4 & 18,7 & 72 \\
\hline 3 & $\begin{array}{l}\text { Finely-dispersed slag }-20 \\
\quad \text { Microsilica }-12 \\
\text { Finely - dispersed cement - } 8\end{array}$ & 13,6 & 14,9 & 20,3 & 14,9 & 85 \\
\hline \multicolumn{7}{|c|}{ At the age of 28 days after hardening under heat-moisture treatment } \\
\hline 4 & Control composition & 14,2 & 16,6 & 23,2 & 29,1 & 50 \\
\hline 5 & Finely-dispersed slag -25 & 13,4 & 17,3 & 20,1 & 20,8 & 65 \\
\hline 6 & $\begin{array}{l}\text { Finely-dispersed slag }-20 \\
\quad \text { Microsilica }-12 \\
\text { Finely - dispersed cement - } 8\end{array}$ & 11,1 & 12,4 & 18.9 & 6,8 & 80 \\
\hline
\end{tabular}

Thermogravimetric studies determined three main sections - fractures of TG curves with an increase in temperature to $1000{ }^{\circ} \mathrm{C}$. This can be caused by the removal of water of various forms of connectivity, by changing the structure of the samples, including phase transitions. Three extremes are determined: at a temperature of $(136-147){ }^{\circ} \mathrm{C}$ - extremum is observed during the evaporation of physically bound water; at $\mathrm{t}=(519-531){ }^{\circ} \mathrm{C}-$ is associated with the removal of chemically bound water; at $t=(822-832){ }^{\circ} \mathrm{C}$ - in connection with the destruction of the hydrated phases of silicates and calcium aluminosilicates. It was also established that the lowest weight loss at the temperature above $820{ }^{\circ} \mathrm{C}$ has had the concrete contained portland cement, fine-dispersed blast-furnace slag, and microsilica and finely-dispersed cement fraction. These results are associated with a lower degree of water in the hydrosilicate phases and confirm their higher strength.

To verify the phase changes in the TG curves, X-ray diagrams of the cement stone of concrete samples were studied. It is determined that the intensity of peaks of low-basic calcium hydrosilicates is very high. The structure of concrete with the indicated mineral modifiers is characterized by a significantly lower intensity of the portlandite peaks, and the degree of hydration of portland cement is $80-85 \%$, significantly exceeding the analogous indicator for the control composition (Table 1). The obtained results of structural analysis indirectly confirm the significantly higher strength of concrete with mineral modifiers characterized by optimal parameters (dispersion, content) at each structural level. 


\section{Conclusion}

The considered theoretical and experimental provisions on the multi-level disperse modification of the RPC composition is one of the main methods for obtaining highperformance concretes with a rational binder content and high performance characteristics. The basic principle of obtaining RPC is to provide a dense homogeneous structure by optimizing the dispersion-granulometric composition at various structural levels and using highly effective superplasticizers (high-range water-reducing admixtures). High density of the structure is achieved by obtaining the maximum dense packages by applying strong particles and grains with high elastic modulus and optimal parameters for each structural level. In this case, it is expedient to use several structural levels of the clinker phase particles and pozzolanization of the matrix in accordance with the optimal formulation.

\section{References}

1. N.M. Azmee, N. Shafiq, Case Stud. Constr. Mater., Ultra-high performance concrete: from fundamental to applications, 9 (2018)

2. H. Yigiter et al., Compos. Part B, Mechanical performance of low cement reactive powder concrete (LCRPC), V.43-8, 2907-2914 (2012)

3. F.F. Leng, Comp. Mater., Destruction of composites with dispersed particles in a brittle matrix, 5, 11-57 (1978)

4. E.G. Velichko, G.S. Belyakova, Building Materials, Physico-chemical and methodological foundations for obtaining multicomponent systems with optimized composition, 3, 27-30 (1995)

5. K.Sobolev, High-Strength Concrete with Low Cement Content (Ph.D.Diss., NIIZhB, Moscow, 1993)

6. G.Batrakov, K.Sobolev, Multicomponent Cement-Based Superplasticized HighStrength Concretes: Design, Properties and Optimization, 695-710 (Fifth canmet/aci international conference on fly ash, silica fume, slag and natural pozzolans in concrete, Milwaukee, 1995)

7. Z.B. Entyn, B.E. Udovich, Multicomponent cements, E 107, 3-76 (NIICsement, Moscow, 1994)

8. I.V. Melikhov, Physicochemical evolution of solid matter (Binom.Know.lab, Moscow, 2009)

9. V.Batrakov, E.Silina, K.Sobolev, N.Zigulev, Recommendations on Production and Application of the High-Strength and Super-High-Strength Highly Dense (NIIZhB, Moscow, 1992)

10. N.A. Hamiruddin, and others, Solid State Phenomena, The effect of different sand gradation with ultra high performance concrete , 280, 476-480 (2018)

11. A. Alsalman, et al., Constr.Build.Mater., Development of ultra-high performance concrete with locally available materials, 133, 135-145 (2017)

12. P. Zhang et al., Constr.Build.Mater., Influence of nano-SiO $\mathrm{O}_{2}$ on properties of fresh and hardened high performance concrete, 148, 648-658 (2017)

13. E.G. Velichko, Structure and the basic properties of building materials (LKI, Moscow, 2014)

14. K.M. Ng et al., Mag. Concr. Res., Studying the production process and mechanical properties of reactive powder concrete: a Hong Kong stud, V 62-9, 647-654 (2010)

15. D. Mostofinejad, M.R. Nikoo, S.A. Hosseini, Constr.Build.Mater., Determination of optimized mix design and curing conditions of reactive powder concrete (RPC), 123, 754-767 (2016) 